JOURNAL OF SYNCHROTRON RADIATION

ISSN 1600-5775

\section{Synchrotron Methods for Energy Materials. $X$-ray Studies on Electrochemical Systems. By Artur Braun. DeGruyter, 2017. Pp. xxviii + 474. Price EUR 79.95, USD 91.99, GBP 65.99, paperback, ISBN 978-3-11-043750-8.}

\author{
Hideto Imai* \\ NISSAN ARC Ltd, 1 Natsushima, Yokosuka, Kanagawa 237-0061, Japan. \\ *Correspondence e-mail: imai@nissan-arc.co.jp
}

Keywords: book review; synchrotron methods; electrochemical systems.

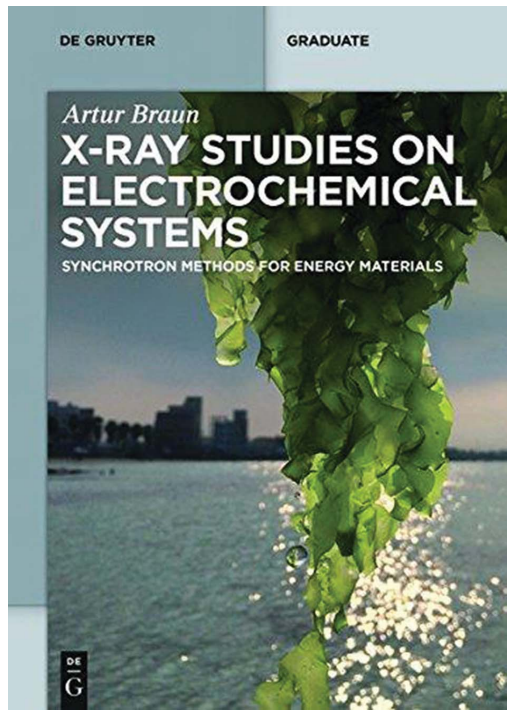

(C) 2018 International Union of Crystallography
This is a good book that is indispensable for students and researchers who are starting $\mathrm{X}$-ray analyses on electrochemical systems and electrochemical energy materials, from a viewpoint of both X-ray specialist and materials researcher.

I have never seen such a book! Many researchers carrying out their experiments are shown in photos! The author of this book, in an unprecedented effort, includes many photographs of researchers conducting their experiments. Planning of the synchrotron experiments at the site, real sampling and setting, know-how of the experimental cells and the experimental equipment, know-how of actual measurements and the data processing are all shown in detail in the photographs. Even those who have never been to a synchrotron radiation facility can learn about the actual experiments as if they were there. It brought back memories of my own experiments at synchrotron radiation facilities as I devoutly read page after page of the book.

Energy devices discussed in this book range from polymer electrolyte fuel cells and solid oxide fuel cells to lithium ion secondary batteries, solar cells, bio-electrodes and so on. This book is structured so as to be able to learn while enjoying 'virtual experiments', aimed at a wide range of readers from undergraduate and graduate students to researchers who want to study this area accordingly. Fundamentals of electrochemical energy devices, their materials, the background and aim of research and experiments, and the basics of data analysis and its interpretation are all described in detail.

An electrochemical energy system is composed of electrodes, catalysts, electrolyte solutions and reactive substances, etc., and functions by causing electrochemical reactions at the electrode surface accompanying electron transfer. These are complex entanglement phenomena in which solids, liquids and gases interplay with each other. In order to analyze these reactions and to evaluate the performance of the electrochemical energy devices, it is necessary to analyze the structure and electronic state of the bulk and surface of the solid electrodes, the structure of the liquid, the solvation structure and diffusion mechanism of ions, and the structure and the electronic state of adsorption species. Also, during operation of electrochemical energy devices, their performances are influenced by external fields such as electrochemical potential, temperature, concentration of chemical species, surrounding humidity, etc. Thus, it is desirable to perform the measurements in operando. In order to satisfy these requirements, various X-ray analysis methods are introduced along with actual examples.

In chapter 2, the basics of structure and electronic state analyses by X-ray spectroscopies are described. XAFS (XANES, EXAFS) using hard X-rays is often used as the main method for analyzing the structure and electronic state of energy device materials. $\mathrm{X}$-ray emission spectroscopy and X-ray photoelectron spectroscopy are also introduced as methods for analyzing the electronic states. The fundamentals of X-ray diffraction, wide-angle and small-angle X-ray scattering, small-angle neutron scattering, X-ray and neutron reflectivity measurements are described in chapter 3 as techniques for analyzing structures of crystalline materials, polymer materials, solutions and their interfaces. Chapter 4 explains the fundamentals of real-space imaging and tomography. In addition to simple transmission imaging in two dimensions, three-dimensional image 
constructions by tomography and three-dimensional chemical imaging by scanning transmission X-ray microscopy are introduced.

In chapter 5, advanced spectroscopy or scattering methods utilizing resonance or X-ray absorption/emission are explained. These are advanced methods for obtaining information on different elements occupying the same crystal site, quantitatively analyzing states with different valences with the same element, and separating information on small-angle scattering for each element. This is particularly effective for analyzing states of doped elements like the positive electrode of a lithium ion battery. In the second half of this book, a huge amount of examples of more complex operando analysis are described, such as operando XRD, XANES, EXAFS, ASAXS and X-ray Raman measurements for lithium ion secondary batteries. The examples are fully loaded with state-of-the-art advanced experiments and make worthwhile reading. In the final part, more practical comments such as precautions against damage due to X-rays in measurements and background processing in spectrum analysis are added.

Such a book as this, focusing on electrochemical systems, and dealing with the wide range of X-ray analysis techniques, has never existed before. It is a recommended book for beginner students to researchers starting X-ray analyses on electrochemical systems at synchrotron radiation X-ray facilities. 